\title{
THE FUNDAMENTAL GROUP OF AN ALGEBRA WITH A STRONGLY SIMPLY CONNECTED GALOIS COVERING
}

\author{
DIANE CASTONGUAY, CLAUDIA CHAIO, AND SONIA TREPODE
}

\begin{abstract}
In this work, we prove that if a triangular algebra $A$ admits a strongly simply connected universal Galois covering for a given presentation then the fundamental group associated to this presentation is free.
\end{abstract}

\section{INTRODUCTION}

Let $A$ be a finite dimensional $k$-algebra over an algebraically closed field, $k$. We denote by $\bmod A$ the module category whose objects are the finitely generated left $A$-modules.

Covering techniques had played an important role in the representation theory of algebras. In [5], K. Bongartz and P. Gabriel introduced the concept of simply connected algebras with the aim of studying representation-finite algebras using covering techniques. Moreover, P. Gabriel reduced the problem of studying the representation theory of representation-finite algebras to the problem of studying the representation theory of its simply connected Galois covering.

In the representation-infinite case covering techniques are more difficult to handle. The case of polynomial growth algebras have been considered by A. Skowroński in [9]. On the other hand, selfinjective algebras have also been studied having into account covering techniques.

In this paper we shall consider triangular algebras, that is, algebras $A=$ $k Q_{A} / I_{A}$ such that $Q_{A}$ has no oriented cycles. More precisely, we are concerned with triangular algebras which admit a strongly simply connected Galois covering for a given presentation $k Q_{A} / I_{A}$ of $A$. The main objective of this paper is to study their fundamental group. When the group $G$ acting on the Galois covering $A$ is free some nice properties hold.

In [6], D. Castonguay and J. A. de la Peña introduced a class of algebras called algebras of the first kind respect to a given presentation $k Q_{A} / I_{A}$ of $A$. They proved that for such algebras the fundamental group $\pi_{1}\left(Q_{A}, I_{A}\right)$ is free. In order to get our main result we prove that if $A$ is a triangular algebra which admits a strongly simply connected Galois covering for a given presentation $k Q_{A} / I_{A}$ then $A$ is of the first kind respect to such presentation. As a consequence we get that if there is a presentation of a triangular algebra which admits a strongly

1991 Mathematics Subject Classification. 16G70, 16G20, 16E10.

Key words and phrases. presentation, fundamental group, Galois covering, strongly simply connected. 
simply connected Galois covering then the fundamental group is free. More precisely, we prove Theorem A.

Theorem A. Let $A$ be a triangular algebra which admits a strongly simply connected Galois covering for a given presentation $k Q_{A} / I_{A}$ of $A$. Then, the fundamental group $\pi_{1}\left(Q_{A}, I_{A}\right)$ is free.

This text is organized as follows. In section 1, we recall some preliminary results and we present two results due to I. Assem, S. Liu and Y. Zhang, (private communication). Section 2 is devoted to prove Theorem A.

\section{Preliminaries}

1.1. Locally bounded categories. Let $k$ be an algebraically closed field. A $k$-category $A$ is a category where for each pair of objects $x, y$ of $A$, the set of morphisms $A(x, y)$ has a $k$-vector space structure and where the composition of morphisms is $k$-bilinear.

We denote by $A_{0}$ the set of objects of the $k$-category $A$.

A $k$-category $A$ is called locally bounded if it satisfies the following conditions.

(1) for each $x \in A_{0}$, the endomorphism algebra $A(x, x)$ is local;

(2) distinct objects in $A_{0}$ are not isomorphic;

(3) for each $x \in A_{0}, \sum_{y \in A_{0}} \operatorname{dim}_{k} A(x, y)<\infty$ and $\sum_{y \in A_{0}} \operatorname{dim}_{k} A(y, x)<\infty$.

A quiver $Q$ is given by two sets $Q_{0}$ and $Q_{1}$ together with two maps $s, e: Q_{1} \rightarrow$ $Q_{0}$. The elements of $Q_{0}$ are called vertices and the elements of $Q_{1}$ are called arrows. We assign to each arrow $\alpha \in Q_{1}$ its start point $s(\alpha)$ and its end point $e(\alpha)$. We say that $Q$ is a locally finite quiver if for any vertex $a \in Q_{0}$ there is a finite number of neighbours of a or equivalently, if there is a finite number of arrows ending at $a$ and a finite number of arrows starting at $a$.

If $A$ is a locally bounded category then there exists a locally finite quiver $Q_{A}$ and an admissible ideal $I$ of the path category $k Q_{A}$ of $Q_{A}$ such that there is an isomorphism $A \simeq k Q_{A} / I$, called a presentation of $A$. The pair $\left(Q_{A}, I\right)$ is called a bound quiver.

The category $A$ is called triangular if the corresponding quiver $Q_{A}$ does not have oriented cycles.

For a basic background on representation theory we refer the reader to $[1,2]$.

1.2. Contours and cycles. We recall some notions from [3]. Let $Q$ be a locally finite quiver. For each vertex $a$ of $Q$, we denote by $\varepsilon_{a}$ the trivial path at $a$. For each arrow $\alpha$ of $Q$, we introduce a new arrow $\alpha^{-1}$ as its formal inverse with $s\left(\alpha^{-1}\right)=e(\alpha)$ and $e\left(\alpha^{-1}\right)=s(\alpha)$.

We shall write the sequences of arrows from left to right.

We recall that a walk in $Q$ is either a trivial path or a sequence $w=c_{1} \cdots c_{r}$ with $c_{i}$ an arrow or the inverse of an arrow. When $s(w)=s\left(c_{1}\right)$ and $e(w)=e\left(c_{r}\right)$ we say that $w$ is a walk from $s(w)$ to $e(w)$.

A non-trivial walk $w=c_{1} \cdots c_{n}$ with $s(w)=e(w)=a$ is called a cycle at a point $a$. Observe that it could be a non oriented cycle. We say that $w$ is 
reduced if $c_{i+1} \neq c_{i}^{-1}$ for all $1 \leq i<n$ and $c_{1} \neq c_{n}^{-1}$; and that $w$ is simple if $s\left(c_{i}\right)$ and $s\left(c_{j}\right)$ are pairwise distinct points, where $1 \leq i, j \leq n$.

By a path $p$ we mean an oriented walk, that is, when for all $i=1, \ldots, n$ in $p$ we have that $p=c_{1} \cdots c_{n}$ where $c_{i}$ are arrows with $e\left(c_{i}\right)=s\left(c_{i+1}\right)$ or they are all inverse of arrows with $s\left(c_{i}\right)=e\left(c_{i+1}\right)$ and in this case we write $p^{-1}=c_{r}^{-1} \cdots c_{1}^{-1}$. Moreover, in the first case, if $s(p)=s\left(c_{1}\right)$ and $e(p)=e\left(c_{r}\right)$ we say that $p$ is a path walk from $s(w)$ to $e(w)$.

We say that the length of a path $p$ is $n$ if $p$ is a sequence of $n$ arrows or $n$ inverse of arrows, that is, $p=c_{1} \cdots c_{n}$ or $p^{-1}=c_{n}^{-1} \cdots c_{1}^{-1}$. We denote it by $\ell(p)$. A non-trivial path $p=c_{1} \cdots c_{n}$ with $s(p)=e(p)$ is called an oriented cycle.

A contour in $Q$ from a vertex $a$ to a vertex $b$ is a pair $(p, q)$ of two non-trivial paths from $a$ to $b$. We say that a contour $(p, q)$ is interlaced if $p=p_{1} p_{2}$ and $q=q_{1} q_{2}$ with $\left(p_{1}, q_{1}\right)$ and $\left(p_{2}, q_{2}\right)$ some contours, or equivalently if the paths $p$ and $q$ have a common point other than $a$ and $b$; and it is called reducible if there exist paths $p=p_{0}, p_{1}, \ldots, p_{n}=q$ in $Q$ from $a$ to $b$ such that, for each $0 \leq i<n$, the contour $\left(p_{i-1}, p_{i}\right)$ is interlaced. In this case, we say that $p$ is reducible to $q$. Otherwise, it is called irreducible.

Next we show a contour $(p, q)$ which is reducible and another which is irreducible.

Example 1.1. (a) Let $(p, q)$ be a contour in $Q$ from $a$ to $b$ as follows

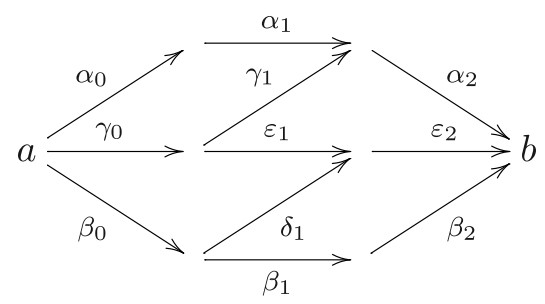

with $p=p_{0}=\alpha_{0} \alpha_{1} \alpha_{2}$ and $q=p_{4}=\beta_{0} \beta_{1} \beta_{2}$. Consider the paths in $Q$ from $a$ to $b, p_{1}=\gamma_{0} \gamma_{1} \alpha_{2}, p_{2}=\gamma_{0} \varepsilon_{1} \varepsilon_{2}$ and $p_{3}=\beta_{0} \delta_{1} \varepsilon_{2}$. Then, $p$ is reducible to $q$, since there is a sequences of paths $p=p_{0}, p_{1}, p_{2}, p_{3}, p_{4}=q$ where $\left(p_{i-1}, p_{i}\right)$ are interlaced for $i=0, \ldots, 4$.

(b) Let $(p, q)$ be a contour in $Q$ from $a$ to $b$ as follows

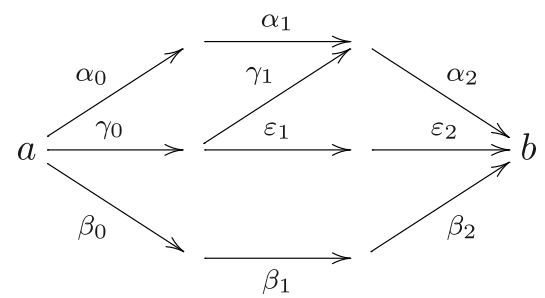

with $p=p_{0}=\alpha_{0} \alpha_{1} \alpha_{2}$ and $q=p_{4}=\beta_{0} \beta_{1} \beta_{2}$. Then, $(p, q)$ is irreducible. 
Let $C=c_{1} \cdots c_{n}$ be a reduced cycle. We write $c_{0}=c_{n}$ and $c_{n+1}=c_{1}$. By a source in $C$ we mean a vertex $a$ having two arrows starting in it. We define $\sigma(C)$ to be the number of sources of $C$. Then $C$ is an oriented cycle if and only if $\sigma(C)=0$; and $\sigma(C)=1$ if and only if there exists a unique $1 \leq i \leq n$ such that $c_{i+1} \cdots c_{n} c_{1} \cdots c_{i}=p q^{-1}$ with $p$ and $q$ some non-trivial paths. In this case, we say that $C$ forms the contour $(p, q)$.

Following [3], we have the following definition of an irreducible cycle.

Definition 1.2. Let $C$ be a simple cycle of $Q$.

(1) If $\sigma(C)=1$, then we say that $C$ is irreducible if it forms an irreducible contour.

(2) If $\sigma(C)>1$, then $C$ is reducible provided that there exists a path $p$ from $a$ to $b(p: a \rightsquigarrow b)$ with $a$ and $b$ vertices of $C$, and two reduced walks $w_{1}$ and $w_{2}$ from $a$ to $b$ such that $C=w_{1} w_{2}, \sigma\left(w_{1} p^{-1}\right)<n$ and $\sigma\left(p w_{2}^{-1}\right)<n$. In any other case, it is said to be irreducible.

Next, we show an example of an reducible cycle.

Example 1.3. Assume that $Q$ is the following quiver:

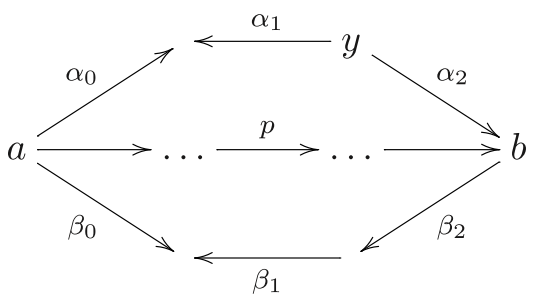

where $p$ is a path in $Q$ from $a$ to $b$. Consider the simple cycle $C=w_{1} w_{2}$ with $w_{1}=\alpha_{0} \alpha_{1}{ }^{-1} \alpha_{2}$ and $w_{2}=\beta_{0} \beta_{1}^{-1} \beta_{2}{ }^{-1}$. Note that $w_{1}$ and $w_{2}$ are reduced walks. Moreover, observe that $C$ is not a contour, and that $x$ and $y$ are the only sources in $C$. Then $\sigma(C)>1$. By Statement (2) of the above definition, we know that $C$ is reducible, since $\sigma\left(w_{1} p^{-1}\right)<2$ and $\sigma\left(p w_{2}^{-1}\right)<2$.

1.3. Homotopy. Let $(Q, I)$ be a connected locally finite bound quiver. An element $\rho=\sum_{i=1}^{n} \lambda_{i} p_{i} \in I(a, b)$, with $\lambda_{i} \in k$ and $p_{i}$ paths from $a$ to $b$ of length at least two, is called a minimal relation of $(Q, I)$ if $n \geq 2$ and for any proper subset $J$ of $\{1,2, \ldots, n\}$ we have that $\sum_{j \in J} \lambda_{j} p_{j} \notin I(a, b)$.

The homotopy relation $\sim$ on the set of walks of $Q$ is the smallest equivalence relation satisfying the following conditions:

(a) If $\alpha: a \rightarrow b$ is an arrow of $Q$ then $\alpha \alpha^{-1} \sim \varepsilon_{a}$ and $\alpha^{-1} \alpha \sim \varepsilon_{b}$.

(b) If $\sum_{i=1}^{n} \lambda_{i} p_{i}$ is a minimal relation then $p_{i} \sim p_{j}$ for all $1 \leq i, j \leq n$.

(c) If $u \sim v$ then $w_{1} u w_{2} \sim w_{1} v w_{2}$ for all walks $w_{1}, w_{2}$ with $e\left(w_{1}\right)=s(u)$ and $s\left(w_{2}\right)=e(v)$.

We say that a cycle is contractible if it is homotopic to a trivial path.

Let $x \in Q_{0}$ be arbitrary. The set $\pi_{1}(Q, I, x)$ of equivalence classes $\bar{u}$ of closed paths $u$ starting and ending at $x$ has a group structure defined by the operation $\bar{u} \cdot \bar{v}=\overline{u \cdot v}$. Since $Q$ is connected then this group does not depend on the choice of $x$. We denote it by $\pi_{1}(Q, I)$ and we call it the fundamental group of $(Q, I)$. 
We write the composition from left to right.

Definition 1.4. We say that a contour $(p, q)$ is a torsion contour in $(Q, I)$ if there is a positive integer $n>1$ such that $\left(p q^{-1}\right)^{n}$ is contractible. We say that the order of the contour $(p, q)$ is $n$ if it is the minimal positive integer $n>1$ such that $\left(p q^{-1}\right)^{n}$ is contractible.

1.4. Natural homotopy. Let $(Q, I)$ be a locally finite bound quiver. Two paths $p, q$ of $(Q, I)$ from a point $a$ to a point $b$ are called naturally homotopic if $p=q$ or if there exists a sequence of paths $p=p_{0}, p_{1}, \ldots, p_{n}=q$ from $a$ to $b$ such that for each $1 \leq i<n$, we can write $p_{i}=u_{i} v_{i 1} w_{i}, p_{i+1}=u_{i} v_{i 2} w_{i}$, where $u_{i}, w_{i}$ are paths and $v_{i 1}, v_{i 2}$ are non-trivial paths appearing in the same minimal relation of $(Q, I)$. It follows immediately that the natural homotopy is an equivalence relation on the set of the paths in $(Q, I)$ which is compatible with the compositions of paths. Moreover, two naturally homotopic paths are homotopic. The converse is not true, there are homotopic paths which are not naturally homotopic as we show in the next example.

Example 1.5. Consider the path algebra given by the quiver $Q$

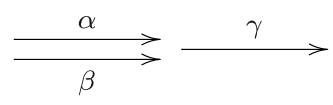

with the ideal $I=\langle\alpha \gamma-\beta \gamma\rangle$. We observe that $\alpha$ and $\beta$ are homotopic in $(Q, I)$ but they are not naturally homotopic. In fact, $\alpha \gamma \sim \beta \gamma$ then $\alpha \sim \beta$.

On the other hand, $\alpha$ and $\beta$ are not naturally homotopic, since they do not have subpaths that form part of a minimal relation.

Definition 1.6 (Assem, Liu, Zhang). Let $C=c_{1} \cdots c_{r}$ be a reduced cycle of $Q$ with $\sigma(C)>0$.

(1) If $\sigma(C)=1$, then $C$ is called naturally contractible if it forms a contour $(p, q)$ with $p, q$ naturally homotopic.

(2) If $\sigma(C)>1$, then $C$ is called naturally contractible if there exists a path (it can be trivial) $p: a \rightsquigarrow b$ with $a$ and $b$ vertices of $C$, and two reduced walks $w_{1}$ and $w_{2}$ from $a$ to $b$ such that $C=w_{1} w_{2}^{-1}, \sigma\left(w_{1} p^{-1}\right)<n$ and $\sigma\left(w_{2} p^{-1}\right)<n$. Moreover both cycles $w_{1} p^{-1}$ and $w_{2} p^{-1}$ are naturally contractible non-oriented cycles.

Next, we show some examples comparing the concept of irreducible or/and contractible cycles.

Example 1.7. (a). We present an example of a reducible contour which is not a contractible cycle. Consider the following quiver

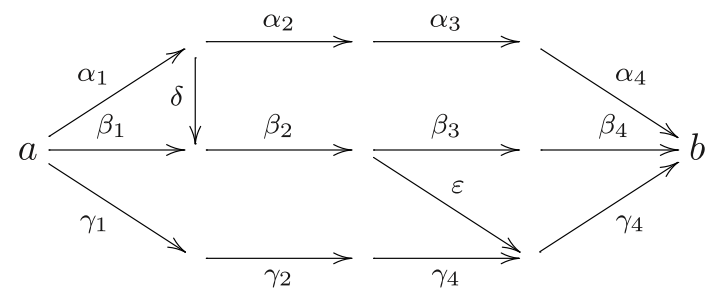


with the relations $\alpha_{2} \alpha_{3}=\delta \beta_{2} \beta_{3} \beta_{4}, \beta_{1} \beta_{2} \varepsilon=\gamma_{1} \gamma_{2} \gamma_{3}$ and $\beta_{3} \beta_{4}=\varepsilon \gamma_{4}$. Let $\alpha=$ $\alpha_{1} \alpha_{2} \alpha_{3} \alpha_{4}$ and $\gamma=\gamma_{1} \gamma_{2} \gamma_{3} \gamma_{4}$. We claim that $(\alpha, \gamma)$ is a reducible contour since it is interlaced. Observe that $(\alpha, \gamma)$ is not a contractible cycle since $\beta_{1} \nsim \alpha_{1} \delta$. Therefore, $\alpha \nsim \gamma$.

(b). Next, we show an example of an irreducible contour which is not a contractible cycle. Consider the contour

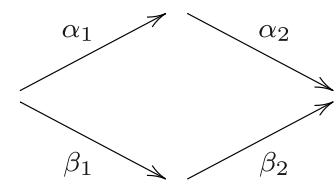

without relations. Then $(\alpha, \beta)$ is irreducible but not a contractible cycle since $\alpha \not \beta$ where $\alpha=\alpha_{1} \alpha_{2}$ and $\beta=\beta_{1} \beta_{2}$.

$(c)$. Let $Q=(\alpha, \beta)$ with $\alpha=\alpha_{1} \alpha_{2}$ and $\beta=\beta_{1} \beta_{2}$ be the contour in $(Q, I)$ as follows

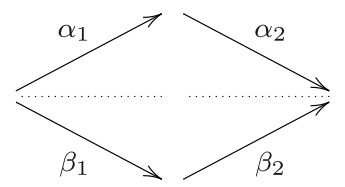

where $I$ is the ideal generated by the relation $\alpha_{1} \alpha_{2}=\beta_{1} \beta_{2}$. The given contour $Q$ is irreducible and moreover is a contractible cycle because $\alpha_{1} \alpha_{2} \sim \beta_{1} \beta_{2}$.

The following result is a direct consequence of the definition.

Lemma 1.8. Let $C=c_{1} \cdots c_{r}$ be a reduced cycle of $Q$. Then

(1) If $C$ is naturally contractible in $(Q, I)$ then $C$ is contractible and reducible in $(Q, I)$.

(2) If there exists $1 \leq i<j<r$ such that $c_{i} \cdots c_{j}$ and $c_{j+1} \cdots c_{s} c_{1} \cdots c_{i-1}$ with $c_{0}=c_{s}$ and $c_{r+1}=c_{1}$ are naturally contractible cycles then $C$ is naturally contractible.

1.5. Strong simply connectedness. Let $\left(Q^{\prime}, I^{\prime}\right)$ and $(Q, I)$ be locally finite bound quivers. We say that $\left(Q^{\prime}, I^{\prime}\right)$ is a convex bound subquiver of $(Q, I)$ if for every points $a, b$ of $Q^{\prime}$, all paths from $a$ to $b$ in $Q$ lie in $Q^{\prime}$ and $I^{\prime}(a, b)=$ $\left(k Q^{\prime}\right) \cap I(a, b)$. We observe that the above convex property does not depends on the presentation.

Let $A$ be a locally bounded category. A full subcategory $B$ of $A$ is called convex if, for any path $x_{0} \rightarrow x_{1} \rightarrow \cdots \rightarrow x_{t}$ in (the quiver of) $A$ with $x_{0}, x_{t} \in B_{0}$ we have that $x_{i} \in B_{0}$, for all $1 \leq i<t$. The category $A$ is called triangular if its quiver $Q_{A}$ contains no oriented cycle.

A triangular locally bounded $k$-category $R \simeq k Q / I$ is said to be simply connected if for every finite convex subcategory $C$ of $R$ and any presentation $C \simeq k Q^{\prime} / I^{\prime}$ we have that the fundamental group of $\left(Q^{\prime}, I^{\prime}\right)$ is trivial, see $[4,5]$ and [8]. 
Definition 1.9. A triangular locally bounded $k$-category $R \simeq k Q / I$ is said to be strongly simply connected if the following two conditions are satisfied:

(1) For every two vertices $x$ and $y$ in $Q$ there are only finitely many paths in $Q$ from $x$ to $y$.

(2) Every finite convex subcategory $C$ of $R$ is simply connected.

Next, we show an example of a simply connected algebra which is not strongly simply connected.

Example 1.10. Let $Q$ be the quiver

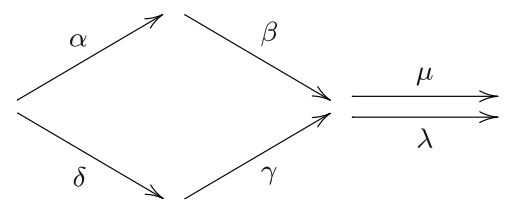

and $I$ be the ideal generated by $\alpha \beta-\gamma \delta$ and $\alpha \beta \lambda-\alpha \beta \mu$.

The algebra $A \simeq k Q / I$ is triangular. Observe that $\Pi_{1}(Q, I)=1$. In fact, since $\alpha \beta \sim \gamma \delta$ then it is left to prove that $\mu \sim \lambda$. We know that $\alpha \beta \mu \sim \alpha \beta \lambda$ then $\beta^{-1} \alpha^{-1} \alpha \beta \mu \sim \beta^{-1} \alpha^{-1} \alpha \beta \lambda$ and therefore $\mu \sim \lambda$.

In order to see that $A$ is simply connected we shall consider all possible presentations of $A$ and prove that for each presentation the fundamental group is trivial. In this case, we have another possible presentation as follows; $\varphi$ : $k Q / I \rightarrow k Q / I^{\prime}$ with $\varphi(I)=I^{\prime}$ that maps $\alpha \rightarrow \alpha, \beta \rightarrow \beta, \delta \rightarrow \delta, \gamma \rightarrow \gamma$, $\mu \rightarrow \mu+a \lambda$ with $a \in k$ and $\lambda \rightarrow \lambda$. Let see that $\Pi_{1}\left(Q, I^{\prime}\right)=1$.

With this new presentation, we have that if we write $\mu^{\prime}=\mu+a \lambda$ then $\mu^{\prime} \sim \lambda$. Indeed,

$$
\begin{aligned}
\alpha \beta \mu^{\prime} & =\alpha \beta(\mu+a \lambda) \\
& =\alpha \beta \mu+a \alpha \beta \lambda \\
& =(a+1) \alpha \beta \mu \\
& \sim(a+1) \alpha \beta \lambda .
\end{aligned}
$$

Hence, we conclude that $\mu^{\prime} \sim \lambda$ and therefore $\Pi_{1}\left(Q, I^{\prime}\right)=1$. Note that any other possible change of presentation is similar to the one analyzed before.

On the other hand, we observe that the algebra $A$ is not strongly simply connected because there is a presentation of $A \simeq k Q / I^{\prime}$ that has a full convex subquiver of the following form

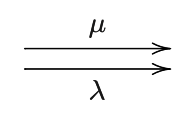

which is not simply connected.

In case we deal with a finite dimensional triangular $k$-algebra $A$ over an algebraically closed field $k$, Definition 1.9 coincides with the formulation if for any presentation $A \simeq k Q / I$ as a bound quiver algebra, the fundamental group $\pi_{1}(Q, I)$ of $(Q, I)$ is trivial, see [4]. 
1.6. Galois coverings. Let $A=k Q / I$ and $A^{\prime}=k Q^{\prime} / I^{\prime}$. Let $F: A^{\prime} \rightarrow A$ be a surjective map induced from a (surjective) quiver map $F_{1}: Q \rightarrow Q^{\prime}$ with $k F_{1}\left(I^{\prime}\right) \subset I$. We say that $F$ is a Galois covering defined by the action of a group of automorphisms $G$ of the bound quiver $\left(Q^{\prime}, I^{\prime}\right)$ if

(1) $F g=F$ for any $g \in G$;

(2) $F_{1}^{-1}(x)=G x^{\prime}, F_{1}^{-1}(\alpha)=G \alpha^{\prime}$ for vertices $x$ in $Q$ and $x^{\prime}$ in $Q^{\prime}$ with $F_{1} x^{\prime}=x$ and arrows $\alpha$ in $Q$ and $\alpha^{\prime}$ in $Q^{\prime}$ with $F_{1} \alpha^{\prime}=\alpha$;

(3) $\mathrm{G}$ acts freely on $A^{\prime}$, that is, if $g \in G$ fixes a vertex in $Q$ then $g=1$.

There is a Galois covering $\widetilde{F}: \widetilde{A}=k \widetilde{Q} / \widetilde{I} \rightarrow A=k Q / I$ defined by the action of $\pi_{1}\left(Q_{A}, I\right)$. For any other Galois covering $F^{\prime}: A^{\prime}=k Q^{\prime} / I^{\prime} \rightarrow A$, there exists a (Galois covering) map $\bar{F}: \widetilde{A} \rightarrow A^{\prime}$ such that $F^{\prime} \bar{F}=\widetilde{F}$. We illustrate the above information in the following diagram:

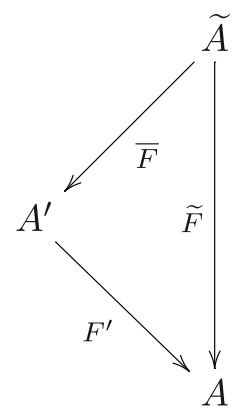

Moreover, the group $\mathrm{H}$ of automorphisms of $\widetilde{A}$ defining $\bar{F}$ is normal in $\pi_{1}\left(Q_{A}, I\right)$ and $\pi_{1}\left(Q_{A}, I\right) / H$ is the group of automorphisms of $A^{\prime}$ defining $F^{\prime}$. Therefore, $\widetilde{F}: \widetilde{A} \rightarrow A$ is called the universal Galois covering of $A$ with respect to $(Q, I)$, see $[8]$.

1.7. On naturally contractible cycles. The following results (theorem and corollary) were proved by I. Assem, S. Liu and Y. Zhang in a private communication. For the benefit of the reader, we present their proofs here.

Theorem 1.11. [Assem, Liu, Zhang] Let $A$ be a connected triangular locally bounded $k$-category. If $A$ is strongly simply connected then for any presentation $A \cong k Q_{A} / I_{A}$ of $A$ we have that every simple cycle of $Q_{A}$ is naturally contractible in $\left(Q_{A}, I_{A}\right)$.

Proof. Let $A \cong k Q_{A} / I_{A}$ be a presentation of $A$. By [3, Theorem 1.3], every irreducible cycle of $Q_{A}$ is an irreducible contour that is naturally contractible in $\left(Q_{A}, I_{A}\right)$. To show that every contour in $Q_{A}$ is naturally contractible in $\left(Q_{A}, I_{A}\right)$, we shall recall the partial order on contours defined in [3] as follows. If $\left(p_{i}, q_{i}\right)$ is a contour from $a_{i}$ to $b_{i}, i=1,2$, then $\left(p_{2}, q_{2}\right) \leq\left(p_{1}, q_{1}\right)$ provided that $\left(p_{1}, q_{1}\right)=\left(p_{2}, q_{2}\right)$ or otherwise $\left(a_{1}, b_{1}\right) \neq\left(a_{2}, b_{2}\right)$ and $a_{1}$ is a predecessor of $a_{2}$ while $b_{1}$ is a successor of $b_{2}$. Let $(p, q)$ be a contour in $Q_{A}$. If $(p, q)$ is minimal, then $(p, q)$ is irreducible, and hence naturally contractible. Suppose that $(p, q)$ is not minimal and every contour less than $(p, q)$ is naturally contractible. If $(p, q)$ is irreducible, then it is naturally contractible. Otherwise there exists a 
sequence of paths $p=p_{0}, p_{1}, \ldots, p_{n}=q$ from $s(p)$ to $e(p)$ such that $\left(p_{i}, p_{i+1}\right)$ is a interlaced contour for all $0 \leq i<n$. Write $p_{i}=u_{1} u_{2}$ and $q_{i}=v_{1} v_{2}$ with $u_{1}, u_{2}, v_{1}$ and $v_{2}$ non-trivial paths such that $e\left(u_{1}\right)=e\left(v_{1}\right)$. Then $\left(u_{1}, u_{2}\right)$ and $\left(u_{2}, v_{2}\right)$ are two contours less than $\left(p_{i}, q_{i}\right)$. Therefore $\left(u_{1}, u_{2}\right)$ and $\left(u_{2}, v_{2}\right)$ are naturally contractible, and hence so is $\left(p_{i}, p_{i+1}\right)$. This implies that $(p, q)$ is naturally contractible.

Let now $C$ be a simple cycle of $Q$. If $\sigma(C)=1$, then $C$ forms a contour $(p, q)$. Hence $C$ is naturally contractible by our claim. Suppose now that $\sigma(C)>1$ and all simples cycles $C^{\prime}$ with $\sigma\left(C^{\prime}\right)<\sigma(C)$ are naturally contractible. Note that $C$ is reducible since $C$ is not a contour. Therefore, there exists a path $u$ from a point $x \in C$ to another point $y \in C$ such that $w_{1} p^{-1}, p w_{2}^{-1}$ are simple cycles with $\sigma\left(w_{1} p^{-1}\right)<\sigma(C)$ and $\sigma\left(w_{2} p^{-1}\right)<\sigma(C)$, where $w_{1}, w_{2}$ are the reduced walks from $x$ to $y$ such that $C=w_{1} w_{2}^{-1}$. By the inductive assumption, the simple cycles $w_{1} p^{-1}, p w_{2}^{-1}$ are naturally contractible. Therefore $C$ is naturally contractible by definition. This completes the proof.

Corollary 1.12. [Assem, Liu, Zhang] Let $A$ be a strongly simply connected locally bounded $k$-category. Then for any presentation $A=k Q_{A} / I_{A}$, every reduced cycle of $Q_{A}$ is naturally contractible in $\left(Q_{A}, I_{A}\right)$.

Proof. Let $n$ be the minimal length of reduced cycles of $Q$. Let $C$ be a reduced cycle. If $C$ is of length $n$, then $C$ is a simple cycle. Hence $C$ is naturally contractible by Theorem 1.11. Assume that the length of $C$ is greater than $n$. If $C$ is a simple cycle, then it is naturally contractible by Theorem 1.11. Otherwise we may assume that $C=C_{1} C_{2}$, where $C_{i}$ are reduced cycles shorter than $C$. So each $C_{i}$ is naturally contractible by the inductive hypothesis. Hence $C$ is naturally contractible by Lemma 1.8 .

1.8. Algebras of the first kind. Let $A$ be a basic connected finite dimensional $k$-algebra with unit, over an algebraically closed field $k$. Consider an epimorphism of $k$-algebras $\nu: k Q_{A} \rightarrow A$ such that $A \simeq k Q_{A} / \operatorname{ker} \nu$. Let $\left(Q_{A}, I_{\nu}\right)$ be a presentation of $A$. For any A-module $X$ we consider the convex full subcategory $A(X)$ of $A$ induced by those vertices in $\operatorname{supp} X$. There is an induced presentation $\nu_{X}: k Q_{X} \rightarrow A(X)$ given by the restriction of $\nu$. An indecomposable $A$-module $X$ is said to be of the first kind with respect to $\nu$ if there is a $\widetilde{B}$ module $Y$ such that $F_{\lambda}^{\prime} Y=X$ for the push-down functor $F_{\lambda}^{\prime}$ associated to the universal Galois covering $F: \widetilde{B} \rightarrow B=A(X)$ with respect to $\nu_{X}$. Furthermore, an algebra $A$ is said to be of the first kind with respect to $\nu_{X}$ if for every vertex $x$ in $Q_{A}$ and every indecomposable direct summand $X$ of $\operatorname{rad} P_{X}$, the module $X$ is of the first kind. We observe that any representation-finite triangular algebra is of the first kind.

If $A$ is a triangular algebra, that is, $A=k Q_{A} / \operatorname{ker} \nu$ where $Q_{A}$ has no oriented cycles then a vertex $x$ in $Q_{A}$ is said to be separating if for the indecomposable decomposition $\operatorname{rad} P_{x}=M_{1} \oplus \cdots \oplus M_{s}$, there is a decomposition into connected components $Q_{A}^{(x)}=Q_{1} \amalg \cdots \amalg Q_{t}$, with $t \geq s$, of the induced full subquiver $Q_{A}^{(x)}$ of $Q_{A}$ with vertices those $y$ which are not predecessors of $x$, such that $\operatorname{supp}_{i}=\left\{y \in Q_{A}: M_{i}(y)=0\right\} \subset Q_{i}$, for $1 \leq i \leq s$. 
Following [4], the algebra $A$ is separated if every vertex $x$ in $Q_{A}$ is separating.

We recall that a $k$-category $A$ is schurian if $\operatorname{dim}_{k} A(x, y) \leq 1$ for every couple of vertices $x, y$ in $Q_{A}$.

Finally, we state the main result given by the authors in [6], since it will be useful for our further considerations.

Theorem([6, Theorem 2.3]) Let $A$ be a triangular algebra of the first kind with respect to the presentation $v: k Q_{A} \rightarrow A$. Consider the universal covering $F: \widetilde{A}_{v} \rightarrow A$. Then, the following conditions hold.

(1) The fundamental group $\pi_{1}\left(Q_{A}, I_{v}\right)$ is free.

(2) If $A$ is schurian then $\widetilde{A}_{v}$ is separated.

For unexplain notions in this subsection we refer the reader to [6].

\section{The main Result}

The aim of this section is to prove our main result. We shall prove that given a triangular algebra $A$ which admits a strongly simply connected Galois covering for a given presentation of $A$ then the fundamental group associated is a free group.

We start this section proving some preliminaries results in order to get the main result.

Lemma 2.1. Let $Q$ be a locally finite quiver and $\gamma$ be a reduced cycle in $Q$. Assume that there exist two vertices $a, b$ in $\gamma$, a path $p$ from $a$ to $b$ and walks $w_{1}, w_{2}$ from a to $b$ such that $\gamma=w_{1} w_{2}^{-1}$, where $w_{1} p^{-1}$ and $w_{2} p^{-1}$ are reduced cycles. Then, exactly one of the following statements hold.

(a) If a is a sink and $b$ is a source in $\gamma$ then $\sigma\left(w_{1} p^{-1}\right)+\sigma\left(w_{2} p^{-1}\right)=\sigma(\gamma)-1$.

(b) If $a$ is not $a$ sink and $b$ is a source in $\gamma$ or either if $a$ is a sink and $b$ is not a source in $\gamma$ then $\sigma\left(w_{1} p^{-1}\right)+\sigma\left(w_{2} p^{-1}\right)=\sigma(\gamma)$.

(c) If neither a is a sink nor $b$ is a source in $\gamma$ then $\sigma\left(w_{1} p^{-1}\right)+\sigma\left(w_{2} p^{-1}\right)=$ $\sigma(\gamma)+1$

Proof. (a) Suppose that $a$ is a sink and $b$ is a source in $\gamma$. Then, we have the following situation:

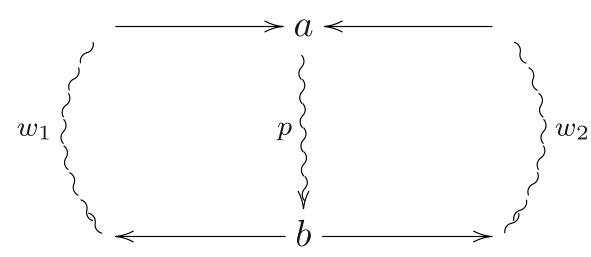

Any source in $\gamma$ which is different from $b$ is a source in $w_{1} p^{-1}$ or a source in $w_{2} p^{-1}$. Clearly, the sources in $w_{1} p^{-1}$ and also in $w_{2} p^{-1}$ are sources in $\gamma$. Thus, $\sigma\left(w_{1} p^{-1}\right)+\sigma\left(w_{2} p^{-1}\right)=\sigma(\gamma)-1$.

(b) Suppose that $a$ is not a sink and that $b$ is a source in $\gamma$. Then, the following situation holds: 


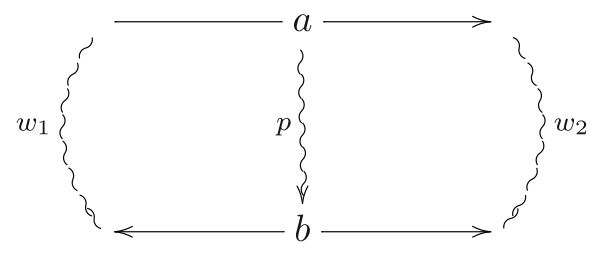

The sources in $\gamma$ which are different from $b$ are either sources in $w_{1} p^{-1}$ or in $w_{2} p^{-1}$. The vertex $b$ is no longer a source in $w_{1} p^{-1}$ neither in $w_{2} p^{-1}$. But, $a$ is a source of one of the cycles $w_{1} p^{-1}$ or $w_{2} p^{-1}$. Moreover, $a$ is a source in both mentioned cycles if $a$ is a source in $\gamma$. Clearly, all sources in $w_{1} p^{-1}$ and all sources in $w_{2} p^{-1}$ which are different from $a$ are sources of $\gamma$. Thus, $\sigma\left(w_{1} p^{-1}\right)+\sigma\left(w_{2} p^{-1}\right)=\sigma(\gamma)$.

Now, suppose that $a$ is a sink and that $b$ is not a source in $\gamma$. Then, we may assume that we have the following diagram:

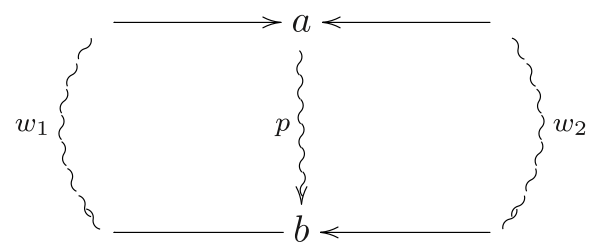

The sources in $\gamma$ coincide with the sources in $w_{1} p^{-1}$ and also with the sources in $w_{2} p^{-1}$. Hence, $\sigma\left(w_{1} p^{-1}\right)+\sigma\left(w_{2} p^{-1}\right)=\sigma(\gamma)$.

(c) Suppose that $a$ is not a sink and $b$ is not a source in $\gamma$. Then, we have one of the following situations:

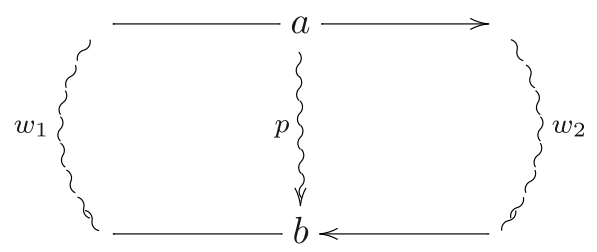

or

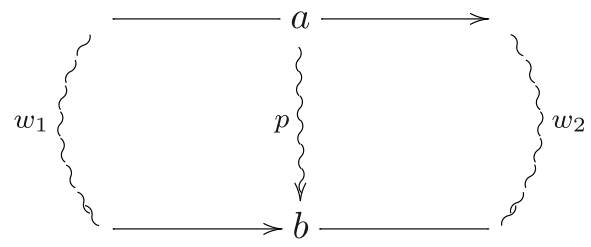

The sources different from $a$ in $w_{1} p^{-1}$ and in $w_{2} p^{-1}$ are sources of $\gamma$. The vertex $a$ is a source in one of the cycles $w_{1} p^{-1}$ or $w_{2} p^{-1}$ (both if $a$ is a source in $\gamma$ ). Then, $\sigma\left(w_{1} p^{-1}\right)+\sigma\left(w_{2} p^{-1}\right)=\sigma(\gamma)+1$.

Proposition 2.2. Let $k Q_{A} / I_{A}$ be a presentation of a triangular algebra $A$ and $F:\left(\widetilde{Q}_{A}, \widetilde{I}_{A}\right) \rightarrow\left(Q_{A}, I_{A}\right)$ be a Galois covering of bound quivers. Let $\gamma$ be a simple cycle in $\widetilde{Q}_{A}$ of the form 


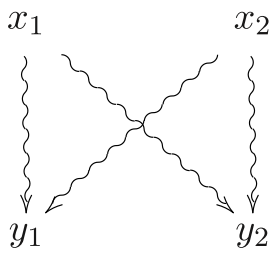

where $x_{i} \rightsquigarrow y_{j}$ are paths for $1 \leq i, j \leq 2, F\left(x_{1}\right)=F\left(x_{2}\right)=x$ and $F\left(y_{1}\right)=$ $F\left(y_{2}\right)=y$. Then, $\gamma$ is irreducible. Consequently, $\gamma$ is not naturally contractible in $\left(\widetilde{Q}_{A}, \widetilde{I}_{A}\right)$.

Proof. Assume that $\gamma$ is reducible. Then, there exist two points $a, b$ in $\gamma$, a path $p: a \rightsquigarrow b$ and walks $w_{1}$ and $w_{2}$ from $a$ to $b$ such that $\gamma=w_{1} w_{2}^{-1}$, $\sigma\left(w_{1} p^{-1}\right)<\sigma(\gamma)=2$ and $\sigma\left(w_{2} p^{-1}\right)<\sigma(\gamma)=2$.

Since $A$ is triangular, the cycles $w_{1} p^{-1}$ and $w_{2} p^{-1}$ are contours and we have that $\sigma\left(w_{1} p^{-1}\right)+\sigma\left(w_{2} p^{-1}\right)=\sigma(\gamma)$. By Lemma 2.1, we have that either $b$ is a source or $a$ is a sink. Therefore, $F(b)=x$ or $F(a)=y$. Since for all vertex $z$ of $\gamma$ we have a path in $Q_{A}$ from $x$ to $y$ going through $F(z)$, then we claim that in both cases, the path $F(p)$ lies in a cycle. In fact, we have $x \rightsquigarrow F(a) \rightsquigarrow F(b)=x$ or $y=F(a) \rightsquigarrow F(b) \rightsquigarrow y$. This yields the desired contradiction, since $A$ is triangular. Hence, $\gamma$ is irreducible.

We define the distance between two points $x, y \in Q_{A}$ as follows:

$$
d(x, y)=\max \{\ell(w) \mid w: x \rightsquigarrow y \text { is a path }\} .
$$

where $\ell(w)$ means the length of the path $w$. Note that the distance between two points in $Q_{A}$ is well defined since $A$ is triangular.

Now we prove a technical lemma useful for further considerations.

Lemma 2.3. Let $k Q_{A} / I_{A}$ be a presentation of a triangular algebra $A$ and $F:\left(\widetilde{Q}_{A}, \widetilde{I}_{A}\right) \rightarrow\left(Q_{A}, I_{A}\right)$ be a Galois covering of bound quivers. Let $(u, v)$, $(u, w)$ and $(v, w)$ be torsion contours from $x$ to $y$ in $Q_{A}$ of minimum distance $d(x, y)$. Let $\gamma$ be a simple cycle in $\widetilde{Q}_{A}$ of the form

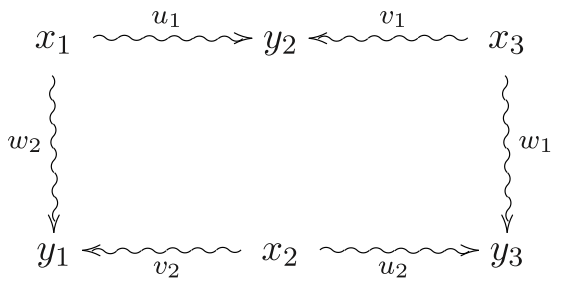

where $u_{1}, u_{2}, v_{1}, v_{2}, w_{1}$ and $w_{2}$ are paths, $F\left(x_{i}\right)=x, F\left(y_{i}\right)=y$ for $i=1,2,3$, $F\left(u_{1}\right)=F\left(u_{2}\right)=u, F\left(v_{1}\right)=F\left(v_{2}\right)=v$ and $F\left(w_{1}\right)=F\left(w_{2}\right)=w$. Then, $\gamma$ is not naturally contractible in $\left(\widetilde{Q}_{A}, \widetilde{I}_{A}\right)$. 
Proof. Assume that $\gamma$ is naturally contractible. Then, there exist vertices $a, b$ in $\gamma$, a path $p$ and walks $\delta_{1}$ and $\delta_{2}$ all of them from $a$ to $b$ such that $\gamma=\delta_{1} \delta_{2}^{-1}$, $\sigma\left(\delta_{1} p^{-1}\right)<\sigma(\gamma)=3$ and $\sigma\left(\delta_{2} p^{-1}\right)<\sigma(\gamma)=3$. Moreover, $\delta_{1} p^{-1}$ and $\delta_{2} p^{-1}$ are naturally contractible reduced cycles. Since for all vertex $z$ in $\gamma$ there is a path from $x$ to $y$ in $Q_{A}$ going through $F(z)$ and $A$ is triangular then we may assume that $F(a) \neq y$ and $F(b) \neq x$. Otherwise, if $F(a)=y$ or $F(b)=x$, the path $F(p)$ lies in a cycle getting a contradiction.

Then, $a$ is not a sink in $\gamma$ and $b$ is not a source in $\gamma$. Since $A$ is triangular, $\widetilde{Q}_{A}$ has no oriented cycles and $\sigma\left(\delta_{i} p^{-1}\right) \geq 1$, for $i=1,2$. Then by Lemma 2.1, we have that $\sigma\left(\delta_{1} p^{-1}\right)+\sigma\left(\delta_{2} p^{-1}\right)=\sigma(\gamma)+1=4$. Therefore, it follows from the fact that $\sigma\left(\delta_{i} p^{-1}\right)<3$, for $i=1,2$ that $\sigma\left(\delta_{i} p^{-1}\right)=2$, for $i=1,2$.

Without loss of generality we may assume that $a$ is a vertex of $u_{1}$. It is not hard to see that either $b$ is in $u_{2}$ or $b$ in $w_{1}$. Indeed if $b \in v_{1}$ then there exists a cyclic permutation of $\delta_{1} p^{-1}$ which is a contour contradicting the number of sources. A similar analysis holds if $b \in v_{2}$.

Now, if $b$ is in $w_{1}$ then we have a diagram as follows

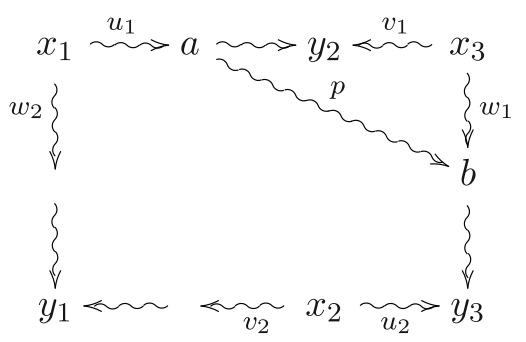

then either one of the naturally contractible cycles $\delta_{1} p^{-1}$ or $\delta_{2} p^{-1}$ is a simple cycle of the form:

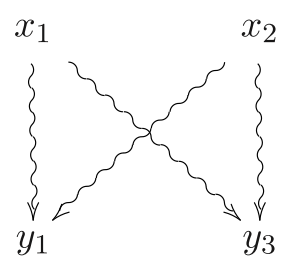

Then, Proposition 2.2 yields the desired contradiction.

Now, if $b$ is in $u_{2}$, then Proposition 2.2 implies that $F(a), F(b) \notin\{x, y\}$. Since $A$ is triangular, there exist paths $q_{1}: x \rightarrow F(a), q_{2}: F(a) \rightarrow F(b)$ and $q_{3}: F(b) \rightarrow y$ such that $u=q_{1} q_{2} q_{3}$.

We claim that $\left(F(p), q_{2}\right)$ is a torsion contour, where $F(p): F(a) \rightarrow F(b)$ is a path in $Q_{A}$. Observe that in $\widetilde{Q}_{A}$, for $i=1,2,3$ there exist paths $q_{i}^{\prime}$ and $q_{i}^{\prime \prime}$ such that $F\left(q_{i}^{\prime}\right)=F\left(q_{i}^{\prime \prime}\right)=q_{i}, u_{1}=q_{1}^{\prime} q_{2}^{\prime} q_{3}^{\prime}$ and $u_{2}=q_{1}^{\prime \prime} q_{2}^{\prime \prime} q_{3}^{\prime \prime}$. Hence, we may assume that $\delta_{1}=\left(q_{1}^{\prime}\right)^{-1} w_{2} v_{2}^{-1} q_{1}^{\prime \prime} q_{2}^{\prime \prime}$ and $\delta_{2}=q_{2}^{\prime} q_{3}^{\prime}\left(v_{1}^{\prime}\right)^{-1} w_{1} q_{3}^{\prime \prime-1}$. By definition $\delta_{i} p^{-1} \sim 1$ for $i=1,2$, since they are naturally contractible reduced cycles. Thus, $F\left(\delta_{i}\right) F\left(p^{-1}\right) \sim 1$ for $i=1,2$. Therefore,

$$
q_{2}^{-1} F(p) \sim q_{2}^{-1} F\left(\delta_{2}\right)=q_{2}^{-1} q_{2} q_{3} v^{-1} w q_{3}^{-1} \sim q_{3} v^{-1} w q_{3}^{-1} .
$$


Since $(v, w)$ is a torsion contour then $v^{-1} w \sim 1$. Hence, we infer that $\left(F(p), q_{2}\right)$ is also a torsion contour because $\left(F(p), q_{2}\right)$ is a contour such that $\left(q_{2}^{-1} F(p)\right)^{2} \sim 1$ getting a contradiction to the minimality of $(v, w)$. Therefore, $\gamma$ is not naturally contractible in $\left(\widetilde{Q}_{A}, \widetilde{I}_{A}\right)$.

Lemma 2.4. Let $k Q_{A} / I_{A}$ be a presentation of a triangular algebra $A$ and $F$ : $\left(\widetilde{Q}_{A}, \widetilde{I}_{A}\right) \rightarrow\left(Q_{A}, I_{A}\right)$ be a Galois covering of bound quivers with $\widetilde{A} \simeq k \widetilde{Q}_{A} / \widetilde{I}_{A}$ strongly simply connected. Let $(u, v)$ be a torsion contour from $x$ to $y$ in $Q_{A}$ of minimum distance $d(x, y)$. Then, there exists a simple cycle $\gamma$ in $\widetilde{Q}_{A}$ of the form

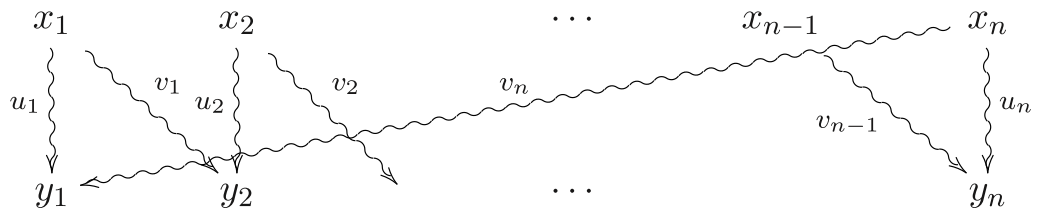

where $n$ is the order of the contour $(u, v), u_{i}: x_{i} \rightsquigarrow y_{i}$ and $v_{i}: x_{i} \rightsquigarrow y_{i+1}$ are paths in $\gamma$ with $y_{n+1}=y_{1}, F\left(u_{i}\right)=u, F\left(v_{i}\right)=v, F\left(x_{i}\right)=x$ and $F\left(y_{i}\right)=y$ for $i=1, \ldots, n$.

Proof. Since $(u, v)$ is a contour of $Q_{A}$ of order $n$ then $\left(u v^{-1}\right)^{n} \sim 1$. Moreover, there exists a reduced cycle $\gamma$ in $\widetilde{Q}_{A}$ of the desired form. It remains to prove that $\gamma$ is simple. Suppose that $\gamma$ is not simple. Then, there exists a vertex $a$ which appears in $\gamma$ at least two times. We analyze all the possible cases where such a vertex can appear in $\gamma$.

Case 1: Consider $a=x_{i}=x_{j}$ with $i \neq j$. Without loss of generality we may assume that $i<j$. Then, in $\widetilde{Q}_{A}$ we have the following situation:

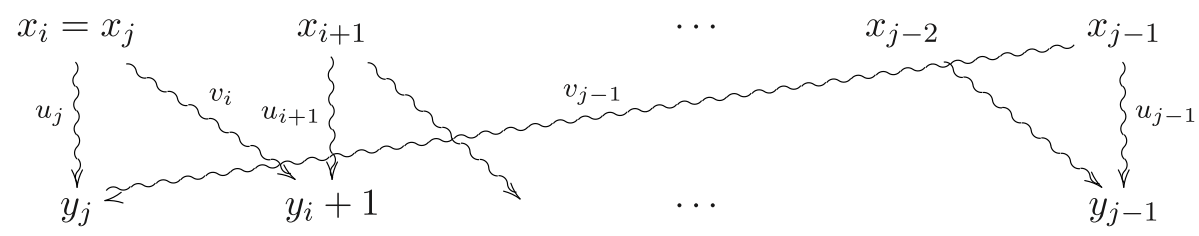

Hence $\left(u v^{-1}\right)^{j-i} \sim 1$, a contradiction to the fact that the order of $(u, v)$ is $n$ and $i<j<n$.

Case 2: Consider $a=x_{i}$ for exactly one $i$. Since $a$ appears at least two times in $\gamma$, then there exist $j \neq i$ and a path from $x_{j}$ to $a$ in $\widetilde{Q}_{A}$.

Note that such a path induces a cycle in $Q_{A}$ since $F(a)=F\left(x_{i}\right)=F\left(x_{j}\right)=x$. This fact leads to a contradiction since $A$ is triangular.

To analyze the others cases we can assume that $F(a) \neq x$. Similarly, we can also assume that $F(a) \neq y$.

Case 3: Consider $a \in u_{i} \cap u_{j}$ (or $a \in v_{i} \cap v_{j}$ ) with $i \neq j$. Since $F(a) \neq x$ there exist two arrows $\alpha: b \rightarrow a$ and $\beta: c \rightarrow a$ such that $u_{i}=w_{i} \alpha w_{i}^{\prime}$ and 
$u_{j}=w_{j} \beta w_{j}^{\prime}$. We may assume that $b \neq c$. Since $F\left(u_{i}\right)=F\left(u_{j}\right)=u$ where $u$ is a simple path and $e(F(\alpha))=F(a)=e(F(\beta))$ we have that $F(\alpha)=F(\beta)$.

On the other hand, $e(\alpha)=a=e(\beta)$. Thus, $\alpha=\beta$ a contradiction to our assumption.

Case 4: Consider $a \in u_{i} \cap v_{j}$. Then, there exists a path $p_{1}: x \rightsquigarrow F(a)$, $p_{2}: F(a) \rightsquigarrow y$ and $q_{1}: x \rightsquigarrow F(a), q_{2}: F(a) \rightsquigarrow y$ in $Q_{A}$ such that $u=p_{1} p_{2}$ and $v=q_{1} q_{2}$.

Without loss of generality, we may assume that $i=1$. Then, in $\widetilde{Q}_{A}$ we have the following situation:

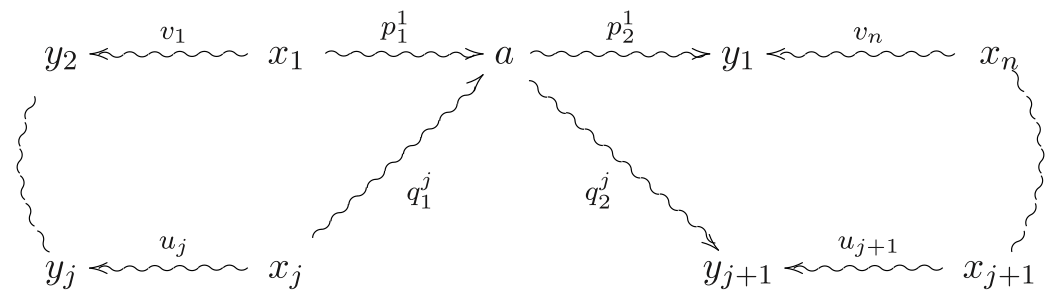

where $p_{1}^{1}, p_{2}^{1}, q_{1}^{j}$ and $q_{2}^{j}$ are paths.

Then, $q_{1}^{-1}\left(u v^{-1}\right)^{j-1} p_{1} \sim 1$ and $p_{2}\left(u v^{-1}\right)^{n-j} q_{2}^{-1} \sim 1$ since $\widetilde{A}$ is strongly simply connected. Thus, $\left(u v^{-1}\right)^{j-1} \sim q_{1} p_{1}^{-1}$ and $\left(u v^{-1}\right)^{n-j} \sim p_{2}^{-1} q_{2}$.

Observe that $1<j<n$. Thus, $2 \leq j \leq n-1$. Since $(u, v)$ is a contour in $Q_{A}$ of order $n$, then we have that $\left(p_{1}, q_{1}\right)$ is a torsion contour contradicting the minimality of the distance of $(u, v)$. Hence, we prove that $\gamma$ is a simple cycle in $\widetilde{Q}_{A}$.

Proposition 2.5. Let $k Q_{A} / I_{A}$ be a presentation of a triangular algebra $A$ and $F:\left(\widetilde{Q}_{A}, \widetilde{I}_{A}\right) \rightarrow\left(Q_{A}, I_{A}\right)$ be a Galois covering of bound quivers with $\widetilde{A} \simeq$ $k \widetilde{Q}_{A} / \widetilde{I}_{A}$ strongly simply connected. If $(u, v)$ is a contour in $Q_{A}$ then $(u, v)$ is not of torsion.

Proof. Assume that there exists $\left(u^{\prime}, v^{\prime}\right)$ a torsion contour in $Q_{A}$. Consider the set

$$
S=\left\{\left(u^{\prime}, v^{\prime}\right) \mid\left(u^{\prime}, v^{\prime}\right) \text { is a torsion contour in } Q_{A}\right\} .
$$

Let $(u, v) \in S$ be a contour in $Q_{A}$ from $x$ to $y$ with minimal distance $d(x, y)$, that is, an element of $S$ starting and ending in points such that their distance is minimal related to all the starting and ending points of the contours in $S$.

We claim that $(u, v)$ is reduced. In fact, otherwise there exist paths $\alpha, \beta, u^{\prime}$ and $v^{\prime}$ in $Q_{A}$ such that $u=\alpha u^{\prime} \beta$ and $v=\alpha v^{\prime} \beta$.

Note that either $\alpha$ or $\beta$ can be trivial paths but not both. Therefore,

$$
u v^{-1} \sim \alpha u^{\prime}\left(v^{\prime}\right)^{-1} \alpha^{-1}
$$

where $\left(u^{\prime}, v^{\prime}\right)$ is a torsion contour. This fact contradicts the minimality of $(u, v)$. Thus the contour $(u, v)$ is reduced.

Consider $\gamma=u v^{-1}$ starting in $x$ and suppose that the order of $(u, v)$ is $n$ with $n>1$. By Lemma 2.4, we have a simple cycle $\widetilde{\gamma}$ in $\widetilde{Q}_{A}$ of the form: 


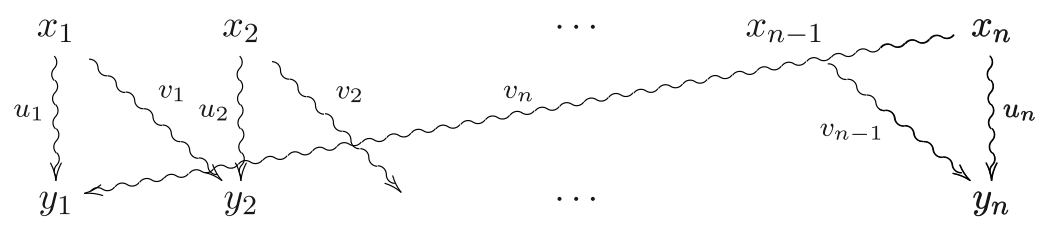

On the other hand, since $\widetilde{A}$ is strongly simply connected, by Corollary 1.12 we know that $\widetilde{\gamma}$ is naturally contractible in $\left(\widetilde{Q}_{A}, \widetilde{I}_{A}\right)$.

Since, $\sigma(\widetilde{\gamma})=n>1$ then there exist a path $p: a \rightsquigarrow b$, and two walks $w_{1}$ and $w_{2}$ from $a$ to $b$ such that $\widetilde{\gamma}=w_{1} w_{2}^{-1}$ and $w_{1} p^{-1}, w_{2} p^{-1}$ are naturally contractible reduced cycles with $\sigma\left(w_{1} p^{-1}\right)<n$ and $\sigma\left(w_{2} p^{-1}\right)<n$.

Therefore, there exist walks $\gamma_{1}, \gamma_{2}$ in $Q_{A}$ from $F(a)$ to $F(b)$ such that $\gamma_{F a}=$ $\gamma_{1} \gamma_{2}^{-1}$. Observe that the cycle $\gamma_{F_{a}}$ is a cycle starting in $F_{a}$, but the underlying graphs of both cycles $\gamma$ and $\gamma_{F_{a}}$ are the same. Then, $F\left(w_{1}\right)=\gamma_{F_{a}}^{r} \gamma_{1}$ and $F\left(w_{2}\right)=\gamma_{F_{a}}^{-s} \gamma_{2}$ for some positive integer $r$ and $s$. Since, $\gamma_{F_{a}}^{n}=F\left(w_{1} w_{2}^{-1}\right)=$ $\gamma_{F_{a}}^{r} \gamma_{1} \gamma_{2}^{-1} \gamma_{F_{a}}^{s}=\gamma_{F_{a}}^{r+s+1}$ we infer that $r+s+1=n$.

Now, since by definition $F(p) \sim F\left(w_{1}\right)=\gamma_{F_{a}}^{r} \gamma_{1}$ and $F(p) \sim F\left(w_{2}\right)=\gamma_{F_{a}}^{-s} \gamma_{2}$ then $F(p) \gamma_{1}^{-1} \sim \gamma_{F_{a}}^{r}$ and $F(p) \gamma_{2}^{-1} \sim \gamma_{F_{a}}^{-s}=\gamma_{F_{a}}^{r+1-n} \sim \gamma_{F_{a}}^{r+1}$.

Next, we analyze all the possible cases where $F(a)$ and $F(b)$ may appear in $(u, v)$.

Case 1: Assume that $F(a)=x$ and $F(b)=y$. Without loss of generality we may assume that $a=x_{1}$ and $b=y_{i+1}$ for some $i \geq 1$ and, moreover that $\gamma_{1}=u$ and $\gamma_{2}=v$. Furthermore, $r \neq 0$ and $s \neq 0$ since otherwise $\sigma\left(w_{1} p^{-1}\right)=1$ or $\sigma\left(w_{2} p^{-1}\right)=1$, a contradiction.

Therefore, $(F(p), u)$ and $(F(p), v)$ are torsion contours in $Q_{A}$. In fact, $F(p) u^{-1}=$ $F(p) \gamma_{1}^{-1} \sim \gamma^{r}$. Since $\gamma$ coincides with $\gamma_{F_{a}}$ and $\gamma^{r}$ is of torsion then $(F(p), u)$ is a torsion contour. With a similar analysis we can get that $(F(p), v)$ is a torsion contour.

Hence in $\widetilde{Q}_{A}$ we have the following reduced cycle $C$

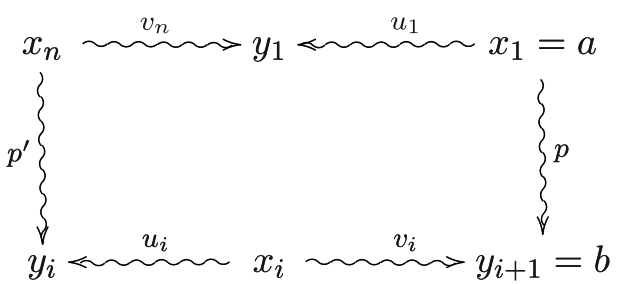

where $p^{\prime}$ is obtained shifting $p$ by the respective group element.

By Corollary 1.12 , we have that $\mathcal{C}$ is naturally contractible in $\left(\widetilde{Q}_{A}, \widetilde{I}_{A}\right)$, a contradiction to Lemma 2.3. Thus, $F(a) \neq x$ or $F(b) \neq y$.

Case 2: Consider $F(a) \neq x$ or $F(b) \neq y$. We may assume that $a$ is a vertex in $u_{1}$. Then, we have to consider two cases if either $b$ is a vertex in $u_{i}$ for some $i \neq 1$ or $b$ is a vertex in $v_{i}$ for some $i$. 
Let first analyze when $b$ is a vertex in $u_{i}$ for some $i \neq 1$, otherwise if $i=1$ then $\sigma\left(w_{2} p\right)=n$ a contradiction.

Since $F(p)$ is a path in $Q_{A}$ from $F(a)$ to $F(b)$ and $A$ is a triangular algebra then there are paths $\delta_{1}: x \rightarrow F(a), \delta_{2}: F(a) \rightarrow F(b)$ and $\delta_{3}: F(b) \rightarrow y$ such that $u=\delta_{1} \delta_{2} \delta_{3}$.

We may assume that $\gamma_{1}=\delta_{2}$ and $\gamma_{2}=\delta_{1}^{-1} v \delta_{3}^{-1}$. Hence,

$$
F(p) \delta_{2}^{-1}=F(p) \gamma_{1}^{-1} \sim \gamma_{a}^{r} .
$$

This implies that $\left(F(p), \delta_{2}\right)$ is a torsion contour in $Q_{A}$, getting a contradiction with the minimality of $(u, v)$.

On the other hand, if $b$ is a vertex in $v_{i}$ for some $i$, then there are paths $\eta_{1}: x \rightarrow F(a), \eta_{2}: F(a) \rightarrow y, \varphi_{1}: x \rightarrow F(b)$ and $\varphi_{2}: F(b) \rightarrow y$, such that $u=\eta_{1} \eta_{2}$ and $v=\varphi_{1} \varphi_{2}$.

We can assume that $\gamma_{1}=\eta_{1}^{-1} \varphi_{1}$ and $\gamma_{2}=\eta_{2} \varphi_{2}^{-1}$. We recall that $F(p) \sim \gamma_{a}^{r} \gamma_{1}$ and $F(p) \sim \gamma_{a}^{r+1} \gamma_{2}$, for some $0 \leq r \leq n-1$. Therefore, replacing $\gamma_{1}$ by $\eta_{1}^{-1} \varphi_{1}$ we get that

$$
\eta_{1} F(p) \varphi_{1}^{-1} \sim \eta_{1} \gamma_{F_{a}}^{r} \gamma_{1} \varphi_{1}^{-1}=\eta_{1} \gamma_{F_{a}}^{r} \eta_{1}^{-1}
$$

and replacing $\gamma_{2}$ by $\eta_{1}^{-1} \varphi_{1}$ we get that

$$
\begin{aligned}
F(p) \varphi_{2} \eta_{2}^{-1} & \sim \gamma_{F a}^{r+1} \gamma_{2} \varphi_{2} \eta_{2}^{-1} \\
& \sim \gamma_{F a}^{r+1} .
\end{aligned}
$$

We conclude that $\left(\eta_{1} F(p), \varphi_{1}\right)$ or $\left(F(p) \varphi_{2}, \eta_{2}\right)$ are torsion contours a contradiction to the minimality of $(u, v)$.

Theorem 2.6. Let $A$ be a triangular algebra admitting a strongly simply connected Galois covering for a given presentation $k Q_{A} / I_{A}$. Then, the fundamental group of $\left(Q_{A}, I_{A}\right)$ is torsion-free.

Proof. Suppose that $\pi_{1}\left(Q_{A}, I_{A}\right)$ is not torsion-free. Let $\bar{\gamma}$ be a torsion element of the group $\pi_{1}\left(Q_{A}, I_{A}\right)$ with $\sigma(\gamma)$ minimum. Observe that $\gamma$ is a cycle in $Q_{A}$ starting at the base point $x$ of the group $\pi_{1}\left(Q_{A}, I_{A}\right)$ and that $\gamma^{n} \sim 1$ for some $n \geq 2$.

Consider $\widetilde{A} \simeq k \widetilde{Q}_{A} / \widetilde{I}_{A}$ the Galois covering of the fixed presentation of $A$. Then, there exists a cycle $\widetilde{\delta}$ in $\widetilde{Q}_{A}$ such that $F(\widetilde{\delta})=\gamma^{n}$.

Now, if $\widetilde{\gamma}$ is irreducible then by [3, Theorem 1.3] we infer that $\widetilde{\gamma}$ is a contour. Furthermore, $\gamma^{n}$ is also a contour. Thus, $n=1$ a contradiction to our assumption. Then, $\widetilde{\gamma}$ is reducible. Since $\widetilde{\delta}$ is not a contour there exists in $Q_{A}$ a path $p: a \rightsquigarrow b$, two walks $w_{1}$ and $w_{2}$ from $a$ to $b$ such that $\widetilde{\delta}=w_{1} w_{2}^{-1}$, $\sigma\left(w_{1} p^{-1}\right)<\sigma(\widetilde{\delta})=n \sigma(\gamma)$ and $\sigma\left(w_{2} p^{-1}\right)<\sigma(\widetilde{\delta})=n \sigma(\gamma)$. Then, there exist in $Q_{A}$ walks $\gamma_{1}$ and $\gamma_{2}$ from $F(a)$ to $F(b)$ such that $\gamma_{F(a)}=\gamma_{1} \gamma_{2}^{-1}$, where $\gamma_{F(a)}$ is a cyclic permutation of $\gamma$. Observe that $F\left(w_{1}\right)=\gamma_{F(a)}^{r} \gamma_{1}$ and $F\left(w_{2}\right)=\gamma_{F(a)}^{-s} \gamma_{2}$ where $r+s+1=n$.

By Lemma 2.1, we have that

$$
\left|\sigma\left(\gamma_{1} F\left(p^{-1}\right)\right)+\sigma\left(\gamma_{2} F\left(p^{-1}\right)\right)-\sigma\left(\gamma_{F(a)}\right)\right| \leq 1 .
$$


Note that $\sigma\left(\gamma_{F(a)}\right)=\sigma(\gamma)$. Suppose that $\sigma\left(\gamma_{1} F\left(p^{-1}\right)\right)=\sigma\left(\gamma_{F(a)}\right)$. Then, $\left|\sigma\left(\gamma_{2} F\left(p^{-1}\right)\right)\right| \leq 1$. Since $A$ is triangular we infer that $\left|\sigma\left(\gamma_{2} F\left(p^{-1}\right)\right)\right|=1$ and therefore $\gamma_{2} F\left(p^{-1}\right)$ is a contour. Moreover, $\gamma_{2} F\left(p^{-1}\right) \sim \gamma_{F(a)}^{s}$. In fact, since $w_{2} p^{-1}$ is contractible due to Corollary 1.12 , so $F(p) \sim F\left(w_{2}\right)$ where $F\left(w_{2}\right)=\gamma_{F(a)}^{-s} \gamma_{2}$. Then $w_{2} p^{-1} \sim 1$. Therefore, $\left.\gamma_{F(a)}\right)^{s} \sim \gamma_{2} F\left(p^{-1}\right)$.

By Proposition 2.5, we know that there is no torsion contour. This means that $s=0$ and $r=n-1$.

On the other hand, since $F\left(w_{1} p^{-1}\right)=\gamma^{r} \gamma_{1} F\left(p^{-1}\right)$ and $\sigma\left(\gamma_{F(a)}\right)=\sigma(\gamma)$ then we have that

$$
\begin{aligned}
\sigma\left(w_{1} p^{-1}\right) & =\sigma\left(F\left(w_{1} p^{-1}\right)\right) \\
& =r \sigma(\gamma)+\sigma\left(\gamma_{1} F\left(p^{-1}\right)\right) \\
& =(n-1) \sigma(\gamma)+\sigma(\gamma) \\
& =n \sigma(\gamma)
\end{aligned}
$$

a contradiction. Then since $A$ is triangular, we conclude that $\sigma\left(\gamma_{1} F\left(p^{-1}\right)\right)<$ $\sigma(\gamma)$. Since $\sigma(\gamma)$ is minimal then $\gamma_{1} F\left(p^{-1}\right)$ is not of torsion.

On the other hand, since $\gamma_{1} F\left(p^{-1}\right) \sim \gamma_{F(a)}^{-r}$ then $r=0$ and $s=n-1$. Since $F\left(w_{2} p^{-1}\right)=\gamma_{F(a)}^{-s} \gamma_{2} F\left(p^{-1}\right)$, we have that

$$
\begin{aligned}
\sigma\left(w_{2} p^{-1}\right) & =\sigma\left(F\left(w_{2} p^{-1}\right)\right) \\
& =s \sigma(\gamma)+\sigma\left(\gamma_{2} F\left(p^{-1}\right)\right) \\
& =(n-1) \sigma(\gamma)+\sigma\left(\gamma_{2} F\left(p^{-1}\right)\right) .
\end{aligned}
$$

Since $\sigma\left(w_{2} F\left(p^{-1}\right)\right)<n \sigma(\gamma)$ this yields that $\sigma\left(\gamma_{2} F\left(p^{-1}\right)\right)<\sigma(\gamma)$. Again by the minimality of $\sigma(\gamma)$ we get that $s=0$. Hence, we prove that $s=0$ and $r=0$. This implies that $n=1$ a contradiction to our assumption, proving the result.

Corollary 2.7. Let $A$ be a triangular algebra which admits a strongly simply connected Galois covering for a given presentation $k Q_{A} / I_{A}$. Then, $A$ is of the first kind.

Proof. By the above theorem, if $A$ admits a strongly simply connected Galois covering for a given presentation $k Q_{A} / I_{A}$ of $A$, then the fundamental group $\pi_{1}\left(Q_{A}, I_{A}\right)$ is torsion-free. Moreover, by $[6,1.8]$ since $F_{\lambda}$ preserves indecomposable modules then $A$ is of the first kind.

By a result due to Castonguay and de la Peña given in [6], it is known that if $A$ is of the first kind respect to a given presentation $k Q_{A} / I_{A}$ of $A$, then the fundamental group $\pi_{1}\left(Q_{A}, I_{A}\right)$ is free.

Therefore we are in position to state our main theorem.

Theorem 2.8. Let $A$ be a triangular algebra which admits a strongly simply connected Galois covering for a given presentation $k Q_{A} / I_{A}$ of $A$. Then, the fundamental group $\pi_{1}\left(Q_{A}, I_{A}\right)$ is free.

\section{REFERENCES}

1. M. Auslander, I. Reiten, S. O. Smalø. Representation Theory of Artin Algebras. Cambridge Stud. in Adv. Math., vol. 36, Cambridge Univ. Press, Cambridge, 1995. 
2. I. Assem, D. Simson, A. Skowroński. Elements of the representation theory of associative algebras. London Math. Soc. Student Texts 65. Cambridge University Press, 2006.

3. I. Assem, and S. Liu. Strongly simply connected Algebras. Journal of Algebra 207, (1998), 449-477.

4. R. Bautista, F. Larion, L. Salmerón. On simply connected algebras. J. London Math. Soc. 27, (2), (1983), 212-220.

5. K. Bongartz, P. Gabriel. Covering spaces in representation theory. Invent. Math. 65, (3), (1981/82), 331-378.

6. D. Castonguay and José A. de la Peña. On the inductive construction of Galois coverings of algebras. Journal of Algebra 263, (1), (2003), 59-74.

7. P. Gabriel. Auslander-Reiten sequences and Representation-finite Algebras. Representation of Algebras, Proc. ICRA II, Ottawa, 1979 in Lectures Notes in Math., Vol 831, Springer, 1980, 1-71.

8. R. Martinez-Villa, J.A. de la Peña. The universal cover of a quiver with relations. Journal of Pure and Applied Algebra 30, (3), (1983), 277-292.

9. A. Skowrońsky. Simply connected algebras and Hoschschild cohomology. Proc. ICRA Iv, Ottawa, 1992, CMS Conf. Proc. Vol 1, pp. 431-447, Amer. Math. Soc., Providence, RI, 1993.

10. A. Skowrońsky. Tame algebras with strongly simply connected Galois coverings. Colloquium Mathematicum 72, no 2, 1997, 335-351.

Instituto de Informática Bloco ImF I, Campus II, Samambaia, Universidade Federal de Goiás, Goiãnia, GO, Brazil.

E-mail address: diane@inf.ufg.br

Centro Marplatense de Investigaciones Matemáticas, Facultad de Ciencias Exactas y Naturales, Funes 3350, Universidad Nacional de Mar del Plata, 7600 Mar del Plata, Argentina

E-mail address: claudia.chaio@gmail.com

Centro Marplatense de Investigaciones Matemáticas, Facultad de Ciencias Exactas y Naturales, Funes 3350, Universidad Nacional de Mar del Plata, 7600 Mar Del Plata, Argentina

E-mail address: strepode@mdp.edu.ar 